\title{
Key workers in the Multi-disciplinary Team
}

\author{
Shaun Stevenson, Charge Nurse, Severalls Hospital, Colchester
}

Today the practice of psychiatry often involves a multidisciplinary team comprising doctors, nurses, psychologists, social workers, occupational therapists, community psychiatric nurses and physiotherapists. Each discipline has its own contribution to make to the planning of care programmes for patients in the context of a team approach.

In the past, decision making was achieved in a totally different way. Primarily the structure was hierarchical, the consultant psychiatrist being the most dominant figure who exerted control and directed the activity at clinical meetings. This autocratic approach directed the focus of attention towards the medical issues of care. Other aspects concerning patients' needs and care were deflected and took less of a priority. Consultation amongst the health care professions was non-existent. With this emphasis, attendance by professionals did not guarantee their comments and opinions being taken into account. This approach gave a very narrow focus to patient care, creating ill feeling within the other disciplines and adversely affecting their job satisfaction. The environment did not exist for individual contribution and personal growth and development was stifled. Often the patient was not consulted about his views and when he was the decisions had frequently already been made.

The modern approach has made major in-roads into breaking down these ways of operating. Today the multidisciplinary team works together to provide optimum care for the individual, decision-making is shared and mutual respect is afforded to each professional group. Each team member is invited to participate in discussion, to express an opinion and to contribute ideas. These are brought together for the benefit of the individual, thus affording him a better service and at the same time greater job satisfaction for professional staff. Decisions are reached through consensus, apart from those that are appropriately made by one discipline, for example nurses on nursing matters. Oocasionally, the final decision will lie with the consultant in overall charge of the patient.

As with any system, there are disadvantages. Firstly, the number of people in the team can create anxiety for staff, particularly juniors and new members, resulting in reluctance to participate and to express views. Secondly, when a key member is absent, decision making may have to be postponed and the progress of the patient will be delayed.
Effective teamwork, a consistent approach to rehabilitation and regular communication between professionals, the patient and relatives, are key factors in the system.

At Severalls Hospital there is an expanding rehabilitation service, using a multi-disciplinary approach. How have we been able to create and establish effective channels of communication and consistency? Initially, all the staff in the team spent a week together in a training programme which also allowed us to get to know each other, to share experiences, to break down barriers and to begin team building. Good personal and professional relationships developed with sharing and exchange of knowledge and skills. This helped to create a respect for, and an acceptance of, the views of colleagues.

In practice, a key worker was attached to a number of residents. The key worker acts as co-ordinator, information gatherer and presenter at review meetings. A record sheet for each clinical review was devised and it is kept in the resident's personal file. Prior to the review the key worker obtains information from the other disciplines involved with the resident; he consults with the patient and areas of care for discussion are highlighted. At the first review meeting the key worker and doctor present a summary of the patient. At subsequent reviews the key worker summarises previous discussions, recaps previously agreed decisions and their follow-up and presents specific areas for current discussion. Other team members are then invited to comment and play an active part in decision making, determining goals and planning programmes of care for the individual. Progress in self-medication is reviewed and finally agreement is reached as to which team members will be responsible for the implementation of agreed plans. Information is then fed back to the patient and his comments and opinions are recorded.

This approach to care benefits both the resident and the staff. The resident is involved in his own care, has access to a variety of disciplines and his progress is regularly monitored. The staff have guidelines and a structured framework with an identified source of information (the review sheet) and they obtain increased job satisfaction through multidisciplinary working, increased involvement and shared responsibility.

This is the second of a series of four papers to be published in the Bulletin on rehabilitation developments at Severalls Hospital. 